\title{
Risk Factors of New Compression Fractures in Adjacent Vertebrae after Percutaneous Vertebroplasty
}

\author{
Myung-Ho Kim¹, Andrew S. Lee², Sang-Hyuk Min¹, Sung-Hyun Yoon ${ }^{1}$ \\ 'Department of Orthopaedic Surgery, Dankook University College of Medicine, Cheonan, Korea \\ ${ }^{2}$ Department of Orthopaedic Surgery, Northwestern University Feinberg School of Medicine, Chicago, IL, USA
}

Study Design: Retrospective study.

Purpose: To evaluate risk factors related to the development of new fractures in adjacent vertebrae after percutaneous ver tebroplasty.

Overview of Literature: Recent reports indicate that undue numbers of new fractures in adjacent vertebral bodies occur after percutaneous vertebroplasty.

Methods: One hundred four of 369 patients who underwent percutaneous vertebroplasty were followed for over 1 year. Fifty-four patients (51.9\%) subsequently suffered from adjacent vertebral fractures. Age, lumbar lordotic angle, sacral slope, pelvic tilt, pelvic incidence, bone mineral density, amounts of cement injected, the restoration of vertebral height, kyphotic angle differences preexisting fracture, and intradiscal cement leakage were noted.

Results: Average bone mineral density was -3.52 in the fracture group and -2.91 in the fracture-free group; the risk of adjacent vertebral fracture increased as bone mineral density decreased ( $p<0.05)$. Intradiscal cement leakage occurred in 18 patients $(33.3 \%)$ in the fracture group, indicating that the risk of adjacent vertebral fracture increased with intradiscal cement leakage. In addition, 36 patients (66.7\%) in the fracture group had a preexisting fracture; thus, the presence of a pre existing fracture was found to be significantly associated with an increased risk of an adjacent vertebral fracture $(p<0.05)$. Higher restoration rates are associated with a greater likelihood of developing adjacent vertebral fractures $(p<0.05)$.

Conclusions: The factors found to contribute significantly to new fractures in adjacent vertebral bodies after percutaneous vertebroplasty were a lower bone mineral density, a greater restoration rate of vertebral height, a pre-existing fracture, and intradiscal cement leakage.

Key Words: Adjacent vertebral fractures, Percutaneous vertebroplasty, Risk factors

\section{Introduction}

As life expectancy increases worldwide, the medical profession inevitably will be more often faced with adult spine disease cases such as osteoporotic vertebral compression fracture. Currently, the therapeutic treatment of choice for vertebral compression fracture is percutaneous vertebroplasty, which proceeds subcutaneous injection of polymethylmethacrylate. This method not only stabilizes the vertebra but also alleviates the pain caused by a vertebral compression fracture. However, recent reports indicate that undue numbers of new fractures in adjacent vertebral bodies occur after percutaneous vertebroplasty. According to Trout

Received Aug 9, 2010; 1st Revised Dec 1, 2010; 2nd Revised Mar 28, 2011; Accepted Apr 8, 2011

Corresponding author: Sang-Hyuk Min, MD

Department of Orthopaedic Surgery, Dankook University College of Medicine,

16-5 Anseo-dong, Cheonan 330-715, Korea

Tel: +82-41-550-3950, Fax: +82-41-556-3238, E-mail: osmin71@ naver.com 
et al. [1], of 432 patients that underwent vertebroplasty, 86 patients (19.9\%) developed 186 new fractures, and 77 of these $(41.1 \%)$ were in vertebrae adjacent to treated vertebrae. Others have suggested that this problem is caused by changes in the load on adjacent vertebra after vertebroplasty [2,3], and have sought relations between risk factors, such as age, bone mineral density, and characteristics and locations of these fractures. This study was undertaken to establish relationships between new fracture development and age, lumbar lordotic angle, sacral slope, pelvic tilt, pelvic incidence, bone mineral density, a preexisting fracture, intradiscal cement leakage, the restoration anterior and middle vertebral heights, and kyphotic angle differences. In addition, we also evaluated a range of risk factors that have been reported to predict the development of vertebral fracture after vertebroplasty.

\section{Materials and Methods}

\section{Materials}

Between January 2006 and December 2009, 369 patients underwent vertebroplasty at Dankook University Hospital (DKUH). A total of 104 patients followed for 1 year were included in this study. At this time, 54 patients (51.9\%) developed new fractures (the fracture group) in an adjacent vertebra and $50(48.1 \%)$ did not (the fracture-free group) (Table 1). Of the 54 patients (51.9\%) in the fracture group, 45 patients $(83.3 \%)$ had a prior history of two fractures and 9 patients of more than two. Age, lumbar lordotic angle, sacral slope, pelvic tilt, pelvic incidence, bone mineral density, preexisting fracture, and intradiscal cement leakage were recorded for all 104 study subjects.

At initial vertebroplasty, 62 patients study subjects had a single vertebral fracture; patients with multiple vertebral fractures were excluded to simplify the analysis. Of these
62 patients, 27 (44\%) developed new fractures in vertebrae adjacent to treated vertebrae, and 35 patients (51\%) did not. The amount of cement injected, the restoration rates of anterior and middle vertebral height and kyphotic angle differences were also measured for the analysis of single vertebral fractures.

The restoration rates of anterior and middle vertebral heights were calculated by taking measurements from lateral radiographs of vertebrae. For these calculations, we assumed that vertebral body prefracture heights were equal to the averages of the heights of adjacent vertebrae, and we then expressed heights before and after vertebroplasty as percentages of presumed normal heights. Kyphotic angle differences were calculated using the angle formed by the upper and lower endplates of vertebral bodies adjacent to fractured vertebrae before and after the procedure (Figs. 1 and 2).

\section{Procedure}

We performed percutaneous vertebroplasty on patients who presented at DKUH complaining of back pain and were found to have a vertebral fracture by magnetic resonance imaging (MRI) or bone scan. One day after the procedure, patients started to walk. Patients were followed up at 2 weeks, 6 weeks, and 3 months post-operation and then every 3 months on an outpatient basis. At 12 months postoperation, patients underwent a second bone scan and were prescribed osteoporotic medication.

\section{Evaluation}

Patients who complained of back pain after initial successful vertebroplasty were evaluated by X-ray, MRI, and bone scan imaging. The evaluation was performed with one of the following criteria: a significant reduction in vertebral

Table 1. Clinical and demographic data for the two study group

\begin{tabular}{llr}
\hline \hline & Fracture group $(\mathrm{n}=54)$ & Fracture-free group $(\mathrm{n}=50)$ \\
\hline Age (yr) & $73.2(59-83)$ & $69.4(60-85)$ \\
Sex & $\mathrm{M}: \mathrm{F}=12: 42$ & $\mathrm{M}: \mathrm{F}=9: 41$ \\
Initial fracture level, $\mathrm{n}(\%)$ & & $8(16.0)$ \\
$\quad$ Thoracic & $8(14.8)$ & $37(74.0)(\mathrm{T} 12, \mathrm{~L} 1 \mathrm{spine})$ \\
T-L junction & $30(55.6)(\mathrm{T} 12, \mathrm{~L} 1$ spine $)$ & $5(10.0)$ \\
Lumbar & $16(29.6)$ & -2.91 \\
BMD (spine) (T-score) & -3.52 & \\
\hline
\end{tabular}

M: Male, F: Female, BMD: Bone mineral density. 
height by X-ray, low attenuation by T1-weighted MRI, high attenuation by T2-weighted MRI, or hot uptake by bone scan. We excluded fractures above the 4th thoracic vertebra from the diagnostic criteria due to a lack of resolution and compression fractures of the sacral vertebra due to difficulties in detection by simple X-ray. We defined the time it took to develop a new fracture as the time lapse from vertebroplasty to adjacent fracture development.

\section{Statistical analysis}

Age, lumbar lordotic angle, sacral slope, pelvic tilt, pelvic incidence, bone mineral density, preexisting fracture, intradiscal cement leakage, the restoration rates of anterior and middle vertebral heights, and kyphotic angle differences were recorded. Baseline comparisons were performed using the $t$-test, and risk factors for new fracture development were analyzed using the logistic regression test. SPSS ver. 15.0 (SPSS Inc., Chicago, IL, USA) was used for all statistical analyses, and $p$-values of $<0.05$ were deemed significant.

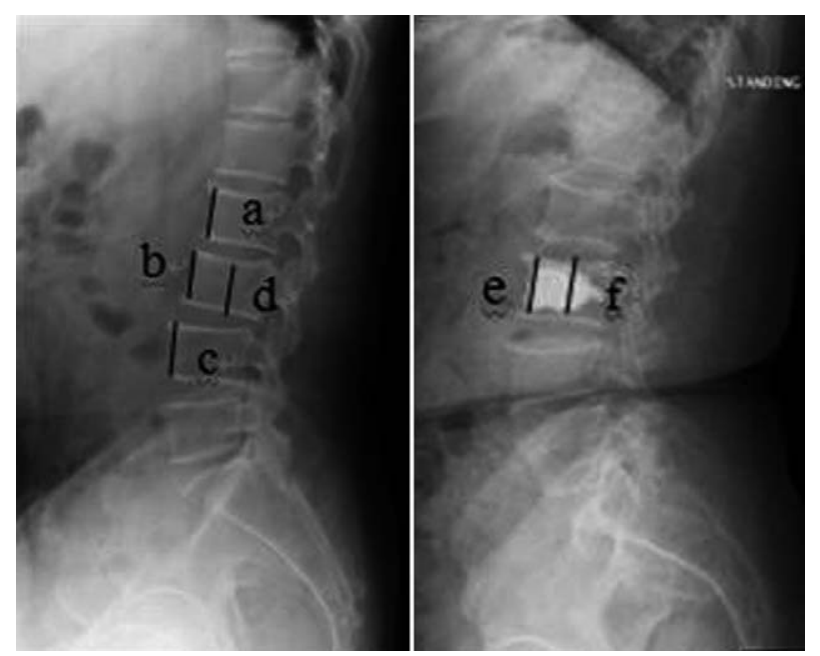

Fig. 1. The method used for determining vertebral body height restoration rate. Vertebral body height before compression fracture $(\mathrm{Y}): \mathrm{Y}=(\mathrm{a}+\mathrm{c}) / 2$. Anterior height restoration $(\mathrm{A}): \mathrm{A}=$ $[(\mathrm{e}-\mathrm{b}) / \mathrm{Y}] \times 100(\%)$. Middle height restoration $(\mathrm{M}): \mathrm{M}=[(\mathrm{f}-$ d) $/ \mathrm{Y}] \times 100(\%)$.

\section{Results}

Average bone mineral density was -3.52 in the fracture group (54 patients), and -2.91 in the fracture-free group (50 patients), indicating that the risk of adjacent vertebral fracture is inversely related to bone mineral density $(p<0.05)$. Intradiscal cement leakage was found in 18 patients $(33.3 \%)$ in the fracture group and in 8 patients $(16 \%)$ of the fracture-free group, indicating that the risk of adjacent vertebral fracture increases with intradiscal cement leakage ( $p$ $<0.05)$. In addition, a preexisting fracture was present in 36 patients $(66.7 \%)$ in the fracture group, and 9 patients $(18 \%)$ in the fracture-free group, which was also significant $(p<$ 0.05 ) (Table 2).

Among the 62 patients with a single vertebral fracture at vertebroplasty, 27 patients developed a new fracture in an adjacent vertebra; these patients had average anterior and middle vertebral height restoration rates of $21.2 \%$ and $22.3 \%$, respectively. For the 35 of the 62 patients $(56 \%)$ who did not develop new fractures, average anterior and middle vertebral height restoration rates were $15.0 \%$ and $15.8 \%$. These results indicate that higher restoration rates are associated with a greater likelihood of developing adja-

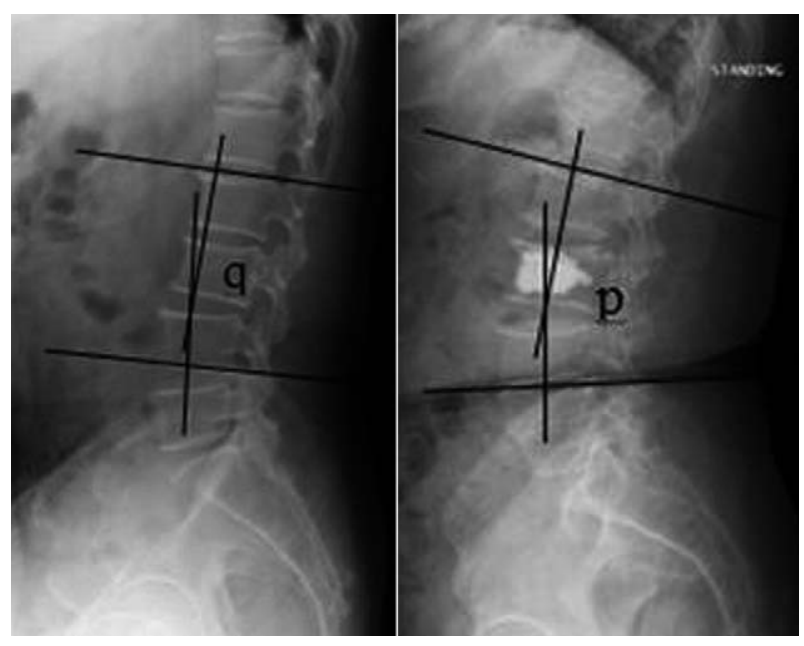

Fig. 2. The method of estimating kyphotic angle differences. Kyphotic angle difference $=\mathrm{p}-\mathrm{q}$.

Table 2. The statistic analysis using Logistic regression test (SPSS ver. 15.0)

\begin{tabular}{lcccccc}
\hline \hline & Fracture group $(\mathrm{n}=54)$ & Fracture-free group $(\mathrm{n}=50)$ & SE & Wald & $p$-value & Exp(B) \\
\hline BMD (spine) (T-score) & $-3.52(-5.5$ to -2.0$)$ & $-2.91(-5.2$ to -1.2$)$ & 0.032 & 4.300 & 0.038 & 0.446 \\
Intradiskal cement leakage & 18 & 8 & 0.654 & 3.949 & 0.047 & 3.949 \\
Pre-existing fracture & 36 & 9 & 0.582 & 13.884 & 0.014 & 4.567 \\
\hline
\end{tabular}

SE: Standard error, BMD: Bone mineral density. 
cent vertebral fractures $(p<0.05)$ (Table 3). However, we found no significant effect between patient age, lumbar lordotic angle, sacral slope, pelvic tilt, kyphotic angle difference, or pelvic incidence and the risk of new vertebral fracture (Table 4).

Nine patients in the fracture group (17.7\%) had experienced vertebral fractures on more than 2 occasions, and for these patients, the average bone mineral density (spine) was -3.84 . This was significantly less than the average density calculated for patients in the fracture-free group $(-2.91, p<$ 0.05) (Table 5).

\section{Discussion}

Osteoporotic vertebral compression fracture is not an uncommon disease in the elderly and can manifest as severe pain, functional deterioration, and limited mobility. Conventional treatments, such as bed rest, bracing, and physical therapy can result in frequent side effects. Furthermore, osteoporotic vertebral compression fractures can cause serious complications when patients undergo surgery, such as vertebral reconstruction or fusion under general anesthesia. Therefore, percutaneous vertebroplasty is considered the treatment of choice to relieve pain and stabilize vertebrae. Although vertebroplasty does have many strong points, it can also lead to a number of complications, and new vertebral compression fractures in adjacent areas are one of the best documented.

The purpose of this retrospective study was to identify the risk factors for new vertebral fractures in a cohort of patients that underwent vertebroplasty. Our retrospective review of charts revealed that 369 patients underwent vertebroplasty at DKUH during the last 3 years, and 104 of these patients who were followed for 12 months were included in this analysis. Of these patients, 52 (51.9\%) developed adjacent vertebral fractures. Several previous studies have noted that time to fracture development after vertebroplasty tends to be short in patients. Chen et al. [4] reported that 20 of 106 patients (18.9\%) developed new fractures in adjacent vertebrae within 24 months of vertebroplasty. Our study shows that of the patients who had developed new fractures in adjacent vertebrae, 31 (57\%) suffered from new fracture development within 6 months of percutaneous vertebroplasty.

Shiraki et al. [5] concluded that low bone mineral density causes degenerative changes in vertebrae, and that low bone

Table 3. Restoration rates in the two study groups (logistic regression; except multi-level fractures)

\begin{tabular}{|c|c|c|c|c|c|c|}
\hline & $\begin{array}{l}\text { Fracture group } \\
\quad(\mathrm{n}=27)\end{array}$ & $\begin{array}{l}\text { Fracture-free group } \\
\qquad(\mathrm{n}=35)\end{array}$ & SE & Wald & $p$-value & $\operatorname{Exp}(B)$ \\
\hline The restoration rate of anterior vertebral height $(\%)$ & 21.2 & 15.0 & 0.044 & 3.956 & 0.047 & 1.092 \\
\hline The restoration rate of middle vertebral height (\%) & 22.3 & 15.8 & 0.114 & 6.097 & 0.014 & 1.756 \\
\hline
\end{tabular}

SE: Standard error.

Table 4. The comparison between the fracture and fracture-free groups

\begin{tabular}{lccr}
\hline \hline & Adjacent fractures group $(\mathrm{n}=54)$ & No adjacent fractures group $(\mathrm{n}=50)$ & $p$-value \\
\hline Age & 73.2 & 69.4 & 0.081 \\
Lodotic angle $\left(^{\circ}\right)$ & 22.9 & 27.5 & 0.094 \\
Sacral slope $\left({ }^{\circ}\right)$ & 32.5 & 33.6 & 0.104 \\
Pelvic tilt $\left({ }^{\circ}\right)$ & 30.8 & 28.5 & 0.117 \\
Pelvic incidence $\left(^{\circ}\right)$ & 63.7 & 62 & 0.167 \\
Kyphotic angle difference & 2.52 & 3.12 & 0.097 \\
\hline
\end{tabular}

Table 5. The comparison between the fracture (fractured over 3 times) and fracture-free group

\begin{tabular}{|c|c|c|c|c|c|c|}
\hline & Adjacent fractures group $(n=9)$ & No adjacent fractures group $(n=50)$ & SE & Wald & $p$-value & $\operatorname{Exp}(B)$ \\
\hline BMD (spine) (T-score) & $-3.84(-5.5$ to -2.0$)$ & $-2.91(-5.2$ to -1.2$)$ & 0.365 & 4.882 & 0.027 & 0.028 \\
\hline Intradiscal cement leakage & 6 & 8 & 0.687 & 5.089 & 0.024 & 4.715 \\
\hline Preexisting fracture & 7 & 9 & 0.732 & 15.976 & 0.021 & 7.657 \\
\hline
\end{tabular}

SE: Standard error, BMD: Bone mineral density. 
mineral density can result in not only vertebral compression fracture but also new vertebral fractures in adjacent vertebrae. Uppin et al. [6] also mentioned that as osteoporosis worsens, patients are more likely to develop new fractures in adjacent vertebrae.

In the present study, average bone mineral density was
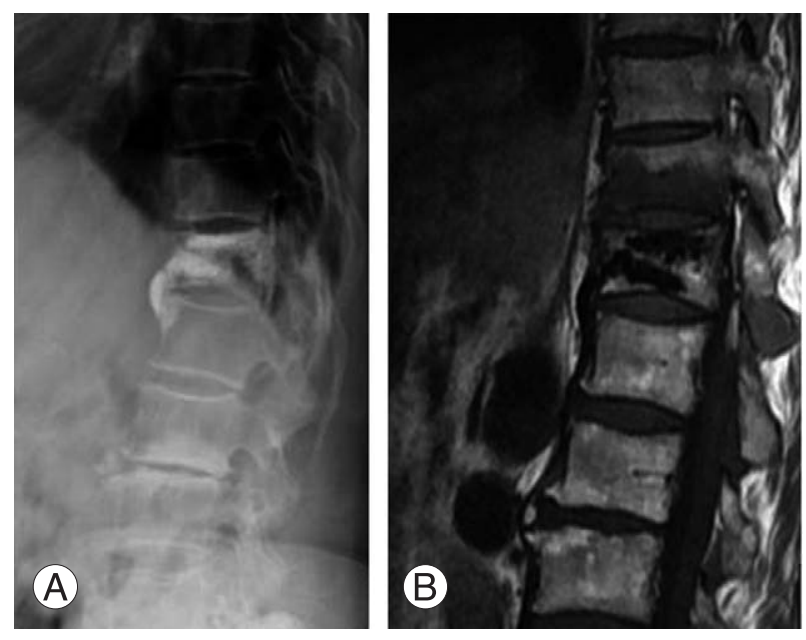

Fig. 3. A 72-year-old female presented with back pain after vertebroplasty of T12 without a previous trauma history. (A) Simple radiograph and (B) magnetic resonance image taken at 6 months postoperatively in a local clinic. A large amount of cement augmentation was observed.
-3.52 in the fracture group and -2.91 in the fracture-free group. Furthermore, 9 patients who had a previous history of vertebral fractures on more than 2 occasions prior to vertebroplasty had a average bone mineral density of -3.84 , which suggests a clear inverse correlation between bone mineral density and the likelihood of a vertebral fracture in adjacent vertebrae $(p<0.05)$. However, factors such as age and gender were found to be unrelated to the risk of fracture, probably because our subjects had similar ages.

It is not clear how new vertebral fractures in adjacent vertebrae occur, though several studies have concluded that cement leakage into the disc increases the risk of these new fractures. Baroud et al. [7] found that the weights of adjacent vertebrae increased by $17 \%$ after percutaneous vertebroplasty. Intradiscal cement works like a pillar. It reduces swelling of the vertebral endplate and vertebral joint mobility, causes swelling of adjacent vertebrae, and increases the risks of adjacent vertebral fracture. According to the functional spine unit (FSU) concept, Belkoff et al. [8] asserted that the volume and distribution of cement injected contribute to changes in vertebral stiffness. Polikeit et al. [9] concluded that the injection of cement into a disc causes an increase in pressure and conformational changes of adjacent vertebral end-plates, thereby increasing the possibility of an adjacent vertebral fracture. Based on the above, we postu-
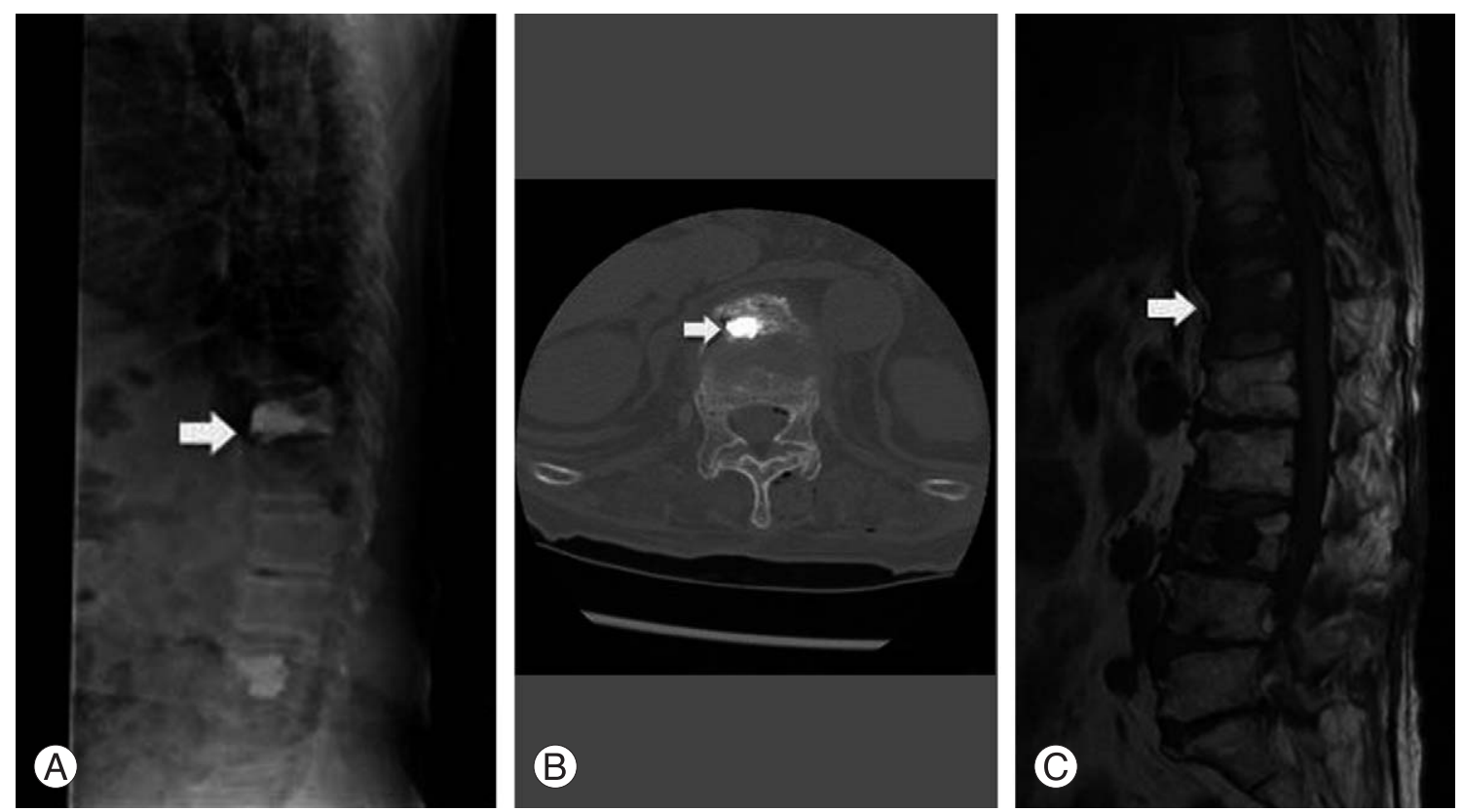

Fig. 4. An 84-year-old female presented with back pain after vertebroplasty. In this case, intra-discal cement leakage was observed. (A, B) After T11 and L3 vertebroplasty, plain radiography and computed tomography showed intradiscal cement leakage in the T11-T12 disc space (arrows). (C) One month after T11 and L3 vertebroplasty, new compression fractures had developed in adjacent T12 (arrow). 
late that an increase in vertebral level due to the injection of cement into a disc increases the weight borne by adjacent vertebra and hence increases the risk of fracture [10]. The present study also shows that higher restoration rates increase the risk of adjacent vertebral fracture. It appears that the restoration of vertebral height increases the bulging of the vertebral end-plate, which is located close to the disc. This results in greater loading of adjacent vertebra, and thus, increases the fracture risk (Fig. 3). Theoretically, the restoration of vertebral height to its original state is considered the ideal. However, in the case of new compression fractures, adequate vertebral height restoration is preferred, rather than restoration to the original height. Lin et al. [11] also suggested the same idea when they found that cement leakage into a disc is one of the most important risk factors for adjacent vertebral fracture after percutaneous vertebroplasty. In addition, Chen et al. [4] also demonstrated a correlation between cement leakage and adjacent vertebral fracture. In the present study, intradiscal cement leakage was found in 18 of 54 patients (33.3\%) in the fracture group and in 8 of $50(16 \%)$ in the fracture-free group, which also demonstrates that intradiscal cement leakage increases the risk of adjacent vertebral fracture $(p<0.05)$ (Fig. 4). Furthermore, it is previously thought that larger cement injection volumes increase the adjacent vertebra fracture risk, but we found no such relation. We believe that difference may be due to fracture location.

Kyphotic angle change after an initial vertebral fracture is also important risk factor of an adjacent vertebral fracture after percutaneous vertebroplasty. Kyphotic angle differ-
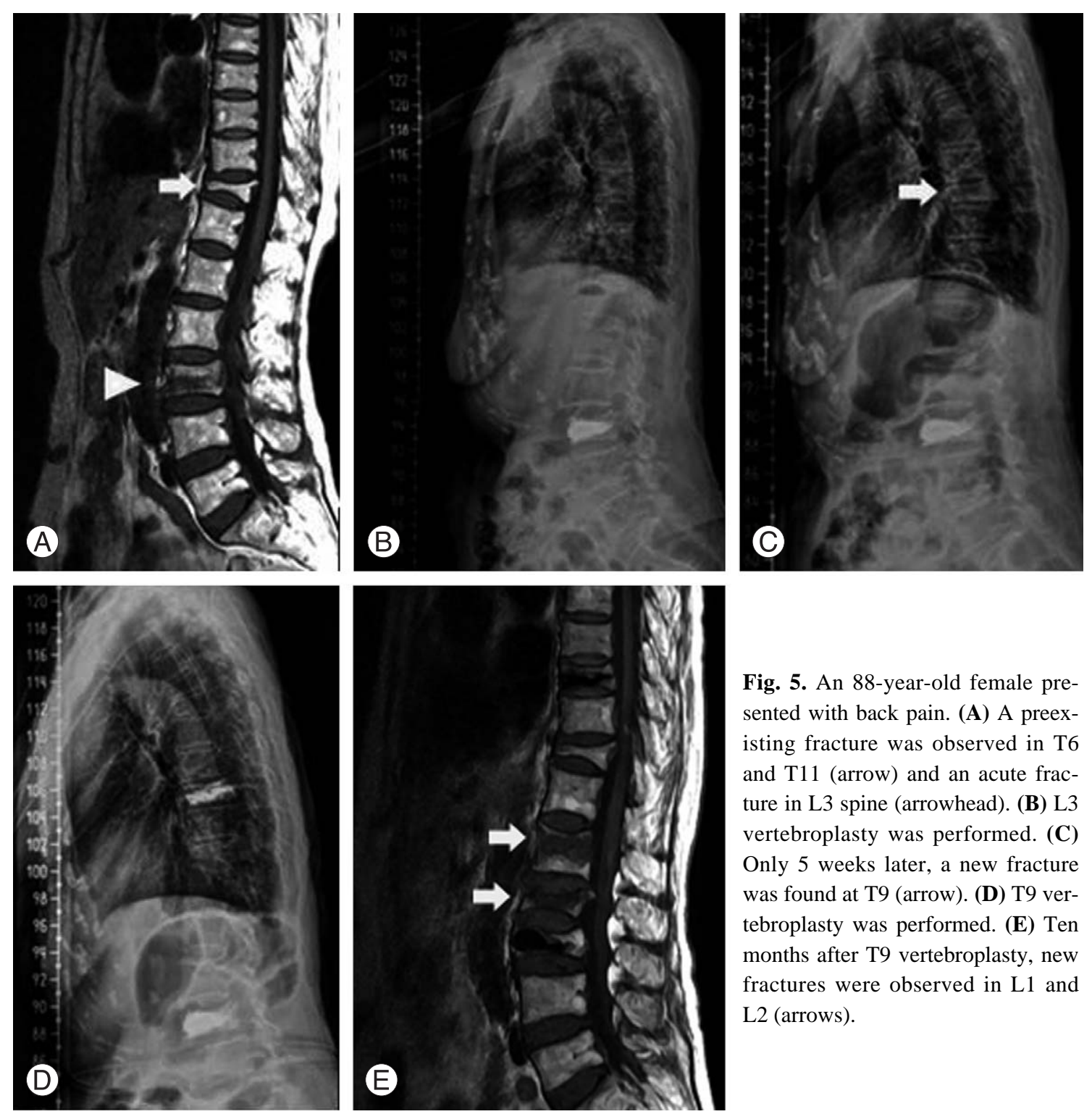

Fig. 5. An 88-year-old female presented with back pain. (A) A preexisting fracture was observed in T6 and T11 (arrow) and an acute fracture in L3 spine (arrowhead). (B) L3 vertebroplasty was performed. (C) Only 5 weeks later, a new fracture was found at T9 (arrow). (D) T9 vertebroplasty was performed. (E) Ten months after T9 vertebroplasty, new fractures were observed in L1 and L2 (arrows). 
ences cause joint stricture and arthritis, in addition to degenerative changes to adjacent vertebrae. Therefore, vertebral bodies should be perfectly aligned to prevent new fractures. Voormolen et al. [12] mentioned that a history of vertebral fractures on more than two occasions is associated with the development of new adjacent vertebral fractures. Our study also shows a significant relationship between new adjacent vertebral fracture development and a prior vertebral fracture history, as $75 \%$ of the fracture group and $44.4 \%$ in the fracture-free group had prior fracture history on more than two occasions $(p<0.05)$ (Fig. 5). However, factors such as lumbar lordotic angle, sacral slope, pelvic tilt and pelvic incidence were not found to be related to the risk of fracture. In addition, when we compaired 9 patients who had a previous history of vertebral fractures on more than 2 occasions prior to vertebroplasty to 50 patient in the fracture free group, the result suggested that low bone mineral density, intradiscal cement leakage, and a preexisting fracture appear to be related to the occurrence of new adjacent vertebral fractures more significantly $(p<0.05)$.

Several studies have reported that the percentage of new adjacent vertebral fracture occurrence after percutaneous vertebroplasty is $20-25 \%$ [13-15]. Our findings strongly confirm those of other studies with respect to the independent risk factors involved, such as, age, bone mineral density and prior history of vertebral fractures, as well as the percutaneous vertebroplasty procedure itself regarding restoration rates and the effect of injecting cement into a disc. These findings indicate that thorough research is required on the relationships between percutaneous vertebroplasty and biomechanical changes in vertebrae so that we can prevent new vertebral fractures.

\section{Conclusions}

In the present study, many risk factors of new vertebral fracture development after vertebroplasty were analyzed. In particular, the development of a new fracture was found to be associated with a lower bone mineral density, greater restoration rate of vertebral height, preexisting fracture, and intradiscal cement leakage. As the incidence of adjacent vertebral fracture after vertebroplasty has frequently been reported, these risk factors should be taken into account.

\section{REFERENCES}

1. Trout AT, Kallmes DF, Kaufmann TJ. New fractures after vertebroplasty: adjacent fractures occur significantly sooner. AJNR Am J Neuroradiol 2006;27:217-23.

2. Berlemann U, Ferguson SJ, Nolte LP, Heini PF. Adjacent vertebral failure after vertebroplasty: a biomechanical investigation. J Bone Joint Surg Br 2002;84:748-52.

3. Schlaich C, Minne HW, Bruckner T, et al. Reduced pulmonary function in patients with spinal osteoporotic fractures. Osteoporos Int 1998;8:261-7.

4. Chen WJ, Kao YH, Yang SC, Yu SW, Tu YK, Chung KC. Impact of cement leakage into disks on the development of adjacent vertebral compression fractures. J Spinal Disord Tech 2010;23:35-9.

5. Shiraki M, Ito H, Fujimaki H, Higuchi T. Relation between body size and bone mineral density with special reference to sex hormones and calcium regulating hormones in elderly females. Endocrinol Jpn 1991;38:343-9.

6. Uppin AA, Hirsch JA, Centenera LV, Pfiefer BA, Pazianos AG, Choi IS. Occurrence of new vertebral body fracture after percutaneous vertebroplasty in patients with osteoporosis. Radiology 2003;226:119-24.

7. Baroud G, Nemes J, Heini P, Steffen T. Load shift of the intervertebral disc after a vertebroplasty: a finite-element study. Eur Spine J 2003;12:421-6.

8. Belkoff SM, Mathis JM, Erbe EM, Fenton DC. Biomechanical evaluation of a new bone cement for use in vertebroplasty. Spine (Phila Pa 1976) 2000;25:1061-4.

9. Polikeit A, Nolte LP, Ferguson SJ. The effect of cement augmentation on the load transfer in an osteoporotic functional spinal unit: finite-element analysis. Spine (Phila Pa 1976) 2003;28:991-6.

10. Kim MH, Min SH, Jeon SH. Risk factors of new compression fractures in adjacent vertebrae after percutaneous vertebroplasty. J Korean Fract Soc 2007;20:260-5.

11. Lin EP, Ekholm S, Hiwatashi A, Westesson PL. Vertebroplasty: cement leakage into the disc increases the risk of new fracture of adjacent vertebral body. AJNR Am J Neuroradiol 2004;25:175-80.

12. Voormolen MH, Lohle PN, Juttmann JR, van der Graaf Y, Fransen H, Lampmann LE. The risk of new osteoporotic vertebral compression fractures in the year after percutaneous vertebroplasty. J Vasc Interv Radiol 2006;17:71-6.

13. Baroud G, Görke U, Beckman L, Steffen T. Physical changes of the vertebral tissue treated with vertebroplasty. XVIII Congress of International Society of Biomechanics; 
2001 Jul 8-13; Zürich, Switzerland. Zürich: Swiss Federal Institute of Technology; 2001. p. 728.

14. Jensen ME, Dion JE. Percutaneous vertebroplasty in the treatment of osteoporotic compression fractures. Neuroimaging Clin N Am 2000;10:547-68.
15. Liebschner MA, Rosenberg WS, Keaveny TM. Effects of bone cement volume and distribution on vertebral stiffness after vertebroplasty. Spine (Phila Pa 1976) 2001;26:154754. 